\title{
Educación media en Colombia, modelos pedagógicos y calidad ${ }^{1}$ \\ High school, quality and pedagogical models. Colombia
}

pp. 21-39

JASMANY LozAno PACHECO²
REC: $30 / 04 / 2020$

ACEP: $15 / 08 / 2020$

\section{Resumen}

Con el presente estudio se procura contribuir a la discusión sobre los resultados de la calidad en función de las Pruebas Saber 11, para estudiantes en Cali y la relación entre las prácticas pedagógicas de los docentes. Para ello, con el uso de estadística descriptiva se explora el modelo pedagógico subyacente en las prácticas docentes, contrastado con los resultados Saber 11. Se decantan diferencias notables en los resultados según rasgos demográficos, sociales, geográficos y escolares que expresan un prisma de posibilidades entre los estudiantes, puesto que en función de sus recursos siguen itinerarios que orientan sus "elecciones" rumbo a instituciones con resultados precarios 0 , por el contrario, a aquellas que cuentan con prestigio académico, estatus socioeconómico, lo cual también coincide con prácticas pedagógicas activas.

Palabras clave: Pruebas Saber 11, calidad de la educación, modelos pedagógicos, desigualdades educativas.

\section{Abstract}

The quality of education is increasingly on the agenda educational. For this, the Saber 11 tests were configured that allow traceability to the educational processes and the factors that affect them. Thus, the present study tries to contribute to the discussion

1. Este artículo es parte del proyecto de investigación sobre Evaluación del impacto de las estrategias pedagógicas y didácticas en las pruebas SABER 11, en Cali.

2. Docente, ICESI. Correo electrónico: jlozanopacheco22@yahoo.es 
on the results of these tests in Cali and the possible relationship between the pedagogical practices of teachers and the results of tests on Saber 11. To do this, a descriptive statistical exercise was carried out with an ICFES database and the pedagogical model underlying teaching practices was explored, contrasted with the Saber 11 results. There are notable differences in the results according to demographic, social, geographic, and school traits that express a prism of possibilities among the students, given that depending on their resources they follow itineraries that guide their "choices" towards institutions with poor results or unlike those that have academic prestige, higher socio-economic status, which also coincides with more active pedagogical practices, denoting probabilities of obtaining better performance on tests.

Keywords: Saber 11 tests, quality of education, pedagogical models, educational inequalities.

\section{Introducción}

La calidad de la educación ha sido motivo de preocupación desde hace ya varias décadas para diferentes actores y autoridades del sector educativo en Colombia. Para optimizar estos procesos es necesario establecer los factores que inciden en los resultados y desempeños educativos y estructurar formas de valorar los avances, logros en los procesos de las instituciones educativas colombianas. Para ello el Instituto Colombiano para la Evaluación de la Educación Superior (ICFES) realiza las Pruebas Saber, las cuales buscan "evaluar, mediante exámenes externos estandarizados, la formación que se ofrece en el servicio educativo en los distintos niveles" (ICFES, 2018), y que permiten valorar los desempeños y el desarrollo de competencias, además de proveer información para los estudiantes, las instituciones educativas y el Estado sobre los procesos educativos y las oportunidades de mejora de la calidad en los mismos. En Colombia las pruebas de Estado vienen promoviendo mejoras en la calidad de la educación a nivel de los estudiantes, las instituciones, en el marco de procesos y estrategias de mejoramiento continuo del sector (ICFES, 2017).

En ese sentido, buscamos caracterizar los resultados en las pruebas Saber 11 de las Instituciones Educativas en Cali (IEC), según algunos atributos sociales, económicos y culturales, considerando los reportes sobre el puntaje global, los puntajes por pruebas y el nivel de desempeño alcanzado por los estudiantes, entre otros. De la misma forma se explora, empíricamente, los resultados en estas pruebas y su relación con las prácticas pedagógicas de los docentes de las IEC.

Así, inicialmente, el documento presenta algunos antecedentes sobre el tema de estudio, seguido de un balance teórico sobre los modelos y estrategias pedagógicas; luego se describen los principales resultados en las pruebas Saber 11 en 2016; después se examinan estos resultados para un grupo de IEC, de acuerdo con el modelo pedagógico que implementan; y, por último, se hacen unas consideraciones finales de los resultados analizados en el documento.

\section{Aspectos que inciden en las pruebas Saber 11}

En las últimas décadas se observan significativos esfuerzos para ampliar el acceso a la educación, reflejados en un aumento en la tasa de matrícula, cercana al $100 \%$ en el nivel primario, y al $80 \%$ en el secundario. Sin embargo, en términos de la calidad de la educación impartida, son notables las diferencias 
entre los colegios en las pruebas Saber 11, atribuibles a factores como: las desigualdades sociales, el perfil de los estudiantes, la pertinencia del currículo, el recurso docente, las estrategias pedagógicas y las condiciones de la infraestructura física y tecnológica de los colegios, entre otros.

A continuación se presenta un balance de algunos estudios sobre los diversos factores que inciden en los desempeños en las pruebas Saber 11. En primer lugar hacemos referencia a los aspectos individuales, seguido de los aspectos familiares y de contexto, para cerrar con las condiciones institucionales o el "efecto escuela".

Así, en un trabajo sobre las pruebas Saber 11, Cárcamo y Mola (2012), siguiendo a Machin y McNally (2005) y a Machin y Pekkarinen (2008), identifican la existencia de una brecha de desempeño académico por género, dinámica que es consistente en diversos estudios, señalando que las mujeres muestran mejor desempeño que los hombres en lenguaje y que estas diferencias son menos importantes en matemáticas o la diferencia es más baja (Cárcamo y Mola, 2012; ICFES, 2017). En términos étnicos, se plantea que los estudiantes pertenecientes a una etnia presentan un rendimiento inferior al de sus pares no étnicos, en las áreas de matemáticas y lenguaje (Meza, 2016).

Los aspectos familiares se examinan a través del nivel socioeconómico, origen geográfico, la formación de los padres como punto de partida y soporte de la trayectoria de los estudiantes. En relación con los niveles socioeconómicos se revela una correlación positiva, pero no causal, entre el puntaje y el nivel socioeconómico del estudiante. Se sugiere que "en promedio, los estudiantes de condiciones menos vulnerables registran puntajes más altos en las cinco pruebas" (ICFES, 2017, p. 61). Un estudio realizado en Bogotá sobre el rendimiento escolar plantea que los estudiantes que están ubicados en los estratos 1 y 2 asisten normalmente a colegios con desempeños bajos (Téllez, 2015). Dinámica igualmente observable a través del índice de Necesidades Básicas Insatisfechas (NBI), encontrando que los resultados más altos del ICFES están asociados con menores índices de NBI; mientras que las mayores carencias de bienes y servicios básicos se relacionan con menores logros educativos en las pruebas (Martínez y Turriago, 2015). En suma, las características socioeconómicas del hogar son un factor clave del rendimiento académico y del ingreso esperado que tendrá el estudiante en su vida adulta (Meza, 2016).

Al analizar la distribución geográfica y espacial de los hogares y de los resultados de las pruebas Saber 11, entre 2005 y 2012, los mejores resultados se concentran principalmente en Bogotá y en los departamentos de Santander, Boyacá y Caldas durante los años 2005-2006; mientras que para el 2012, Cundinamarca, área metropolitana de MedeIlín, Quindío, Risaralda y Norte de Santander evidencian importantes avances (Martínez y Turriago, 2015). En efecto, si un estudiante se educa en un lugar distinto a Bogotá tiende a tener un desempeño inferior en los componentes de matemáticas y lenguaje; más difícil aún si los estudiantes residen en zonas rurales (Cárcamo y Mola, 2012; Rozo, 2017).

Otros estudios en el contexto familiar destacan cómo el nivel de escolaridad de los progenitores afecta las pruebas; un nivel bajo de formación académica muestra mayor probabilidad de ubicarse en un nivel bajo de desempeño (Meza, 2016; Téllez, 2015). Por su parte, Llanos et al. (2016), a través de un análisis multinivel, encuentran efectos positivos 
del acompañamiento de la madre a las actividades educativas de sus hijos, dado que fomenta buenos hábitos de estudio y genera una mayor motivación hacia el logro escolar.

En tercer lugar, la literatura también subraya el "efecto escuela" sobre las pruebas Saber 11. Tal es el caso de la jornada escolar a la que asisten los estudiantes, dado que las instituciones con única jornada obtienen mayores puntajes en las pruebas que quienes van a colegios con doble jornada (Castro et al., 2016). Otros análisis destacan que los estudiantes que proceden de colegios privados obtienen, en promedio, mejores resultados que sus pares de colegios públicos (Martínez y Turriago, 2015). Diferencias que tienden a ser más significativas en favor de los centros privados, en especial en el área de inglés.

Así mismo, se evidencia la existencia de una convergencia de atributos en los resultados entre colegios públicos y privados, originadas por las diferencias de perfil de los estudiantes, específicamente por las diferencias en dotación de recursos escolares, seguidas de las familiares e individuales (Castro et al., 2016). Igualmente, el capital humano de las instituciones revela que los docentes con educación superior tienden a favorecer mejores resultados en las pruebas del ICFES (Martínez y Turriago, 2015). Otros estudios llaman la atención sobre factores como los efectos en las pruebas desde las prácticas y los estilos pedagógicos. Una investigación en el departamento del Huila encontró que

la tendencia de los docentes de las áreas de ciencias naturales y matemáticas es la del desarrollo de su actividad pedagógica desde el estilo práctico, pero difieren en el tipo de práctica, que para ciencias naturales es de tipo expositivo (clase magistral), mientras que para el área de matemáticas es de tipo constructivo (taller). (Trujillo, 2015, p. 83)
En suma, esta revisión - no exhaustivasobre algunos factores que inciden en Colombia en las Pruebas Saber 11, revelan la presencia de factores individuales (sexo, etnia, etc.) y factores familiares (estrato, ingresos de los padres, actividad laboral del estudiante, capital educativo del hogar) al igual que factores contextuales (lugar de residencia, etc.) y factores institucionales (características del colegio, formación de los docentes, prácticas pedagógicas, entre otros).

Sin embargo, a pesar de la evidente importancia del "efecto escuela", se manifiesta que se han hecho pocos estudios sobre el lugar de la pedagogía y su relación con los desempeños en las Pruebas Saber 11.

En este sentido, este balance nos invita a explorar la incidencia de las estrategias pedagógicas activas adoptadas por algunos colegios de Cali, y su efecto sobre los desempeños académicos de sus estudiantes en las Pruebas Saber 11 en 2016.

\section{M arco conceptual}

Los cambios culturales, sociales y económicos que han experimentado nuestras sociedades en las décadas recientes, han incidido de manera significativa en el sistema educativo, evidenciado en una masificación global, desigual acceso a los diferentes niveles de educación y tasas normalmente elevadas de deserción (Ezcurra, 2011). Este proceso transforma el perfil de los docentes y de los estudiantes, muta la relación entre ellos y de ellos con el conocimiento. Estos cambios han configurado una "crisis" del sentido "tradicional" de la escuela, de los dispositivos de organización social, de control y de las tecnologías educativas. En consecuencia, se visibilizan nuevos problemas y demandas a la escuela: 
El examen y la evaluación ya no pueden cumplir una función selectiva, sino estrictamente pedagógica y los problemas de aprendizaje ya no se resuelven por la vía fácil y corta de la repetición y la exclusión. Lo mismo puede decirse de los "problemas de conducta y disciplina". (Tenti, 2010, p. 39)

Un antecedente importante en este proceso se deriva de la constitución de un campo del saber relacionado con el sentido, estrategias y la reflexión sobre los procesos de enseñanza-aprendizaje. Sin embargo, este campo tiene un estatuto que no ha sido ajeno a la discusión y a diferentes tensiones disciplinares, incluso la aparición de concepciones educativas que niegan su relevancia, afirmando que "la pedagogía sería un aprendizaje del cual se podía prescindir" (Ríos, 2008, p. 244). Es solo a inicios del siglo XX cuando la reflexión pedagógica se separa de la filosofía y se va constituyendo como un campo disciplinario, con pretensiones de cientificidad, a partir del impulso generado por los promotores del movimiento pedagógico más importante del siglo XX en Colombia autodenominado: la escuela nueva (Flórez, 2005, p. 158). De esta forma, en el contexto del surgimiento de las Normales en Colombia, se señalaba que:

Durante los años 30 del siglo XX, existían claras intenciones de constituir la pedagogía como una disciplina autónoma y central de las reflexiones sobre la escuela, la enseñanza y los métodos de enseñanza, y a la vez, en el filtro por medio del cual se apropiarían otras disciplinas científicas interesadas en los asuntos de la escuela. (Ríos, 2008, p. 244)

\section{Sobre los modelos pedagógicos}

La reflexión pedagógica es un campo significativo para entender los contextos, la práctica docente y los nuevos desafíos educativos. Sin embargo, aspirar a tener una definición unívoca del concepto es un tema difícil, dada la complejidad de los procesos y las dinámicas sociales y educativas que esta categoría entraña y que desborda los propósitos de este artículo. A su carácter polisémico le subyacen diferentes procesos que implican conocer el modelo de sociedad en el que se conciben y el carácter prescriptivo en relación con la sociedad que se pretende construir. En este sentido, algunos autores plantean que estos son construcciones o esquemas mentales, que se configuran en unidades de sentido para entender, explicar y comprender las realidades sociales en las que operan y desde los cuales se construyen concepciones sobre la educación y la práctica pedagógica (Flórez, 2005). Mientras otros lo entienden como el "conjunto de conocimientos y teorías estructuradas sobre la educación, y la práctica cotidiana de los procesos que le atañen" (Vives, 2016, p. 41).

Not (1994) señala que desde alrededor del siglo XVIII se visibilizan al menos dos perspectivas pedagógicas, una en la que se busca enseñar, instruir y formar, se le dirige, modela y equipa al sujeto; y otra que define que el sujeto lleva en sí mismo los medios para lograr su desarrollo y por lo tanto toda acción que intervenga desde el exteriorno hará sino deformarlo y obstaculizarlo. Al respecto, las teorías pedagógicas presentan múltiples clasificaciones, por ejemplo se abordaba la reflexión sobre las escuelas pedagógicas, divididas por una línea entre la escuela vieja - pasiva - o bancaria, como lo enuncia Paulo Freire, y modelos de pedagogías progresistas, vinculadas a una escuela nueva - activa-. De allí que algunos autores proponen la existencia de varios modelos: el tradicional, conductista, experiencial o naturalista, constructivista, social (Flórez, 2005). Otros sugieren que estos se pueden organizar en tendencias racionalistas o tradicionales, románticas 0 
escuela nueva y marxistas o pedagogías críticas (Vives, 2016). Además, se propone la emergencia de algunas pedagogías desde el sur.

Un intento de síntesis de estas posiciones sostiene que en la historia de la educación solo han existido dos grandes modelos con múltiples matices; primero el de tipo heteroestructurante, academicista, verbalista, donde el estudiante es pasivo y receptor, la creación del conocimiento se realiza por fuera del salón de clase y que la función esencial de ella es transmitir la cultura humana a las nuevas generaciones (de Zubiría, 2006). En efecto, se expresa aquí una visión en donde los actores son simples reflejos del mundo en el que viven y básicamente reproducen las estructuras sociales, económicas y culturales de su época. Perspectiva que respondería a la enseñanza tradicional, transmisora o conductista. En contraste, aparece el enfoque autoestructurante, en donde el niño tiene las condiciones necesarias para "jalonar" su propio desarrollo. La educación es un proceso autónomo, donde las intervenciones externas limitan el desarrollo (de Zubiría, 2006). En este sentido, se favorece la acción del individuo y los sentidos que este les asigna a sus experiencias y el poder de orientarse más allá de los condicionamientos históricos y culturales. En esta línea se ubicarían los modelos de la escuela nueva o constructivista.

En tercer lugar se propone la posibilidad de crear un modelo - el interestructuranteque procure ser una síntesis dialéctica de los dos modelos precedentes, lo cual implica que

hay que reconocer que el conocimiento se construye por fuera de la escuela, pero que es reconstruido de manera activa e interestructurada a partir del diálogo pedagógico entre el estudiante, el saber y el docente, y que para que ello ocurra es indispensable contar con la mediación adecuada de un maestro, que favorezca de manera intencionada, significativa y trascedente el desarrollo integral del estudiante. Así se postula una educación que no puede estar centrada en el aprendizaje, como desde hace siglos ha creído la escuela, sino en el desarrollo. (de Zubiría, 2006, p. 194)

Esta última apuesta conceptual es la que usamos como marco interpretativo de este estudio, procurando describir la forma como emergen los modelos hetero, auto e interestrucurante en la práctica de los docentes de las instituciones educativas en Cali. Para operacionalizar la práctica pedagógica relacionada con cuestiones fundamentales asumimos criterios como:

¿Con qué propósito o fines se desarrolla el proceso educativo? ¿Quiénes son los sujetos del acto educativo? ¿Cuál es el rol del maestro, del estudiante y del conocimiento? ¿Cuál es la relación entre ellos? ¿Cuáles son los conocimientos y saberes que se deben enseñar y aprender? ¿Cuáles son las diferentes formas o estilos de aprendizaje? ¿Cómo se evalúa 0 se valora el aprendizaje? ¿Con qué recursos humanos, físicos y materiales se apoya el proceso educativo? (Vives, 2016; de Zubiría, 2006).

Hay que precisar que los modelos pedagógicos, en la forma como se abordan en este estudio, son construcciones que fungen como tipos ideales que buscan contribuir a la comprención de la acción docente, influenciada por toda especie de matices y desviaciones esperados de la acción y que, por lo tanto, es poco probable encontrar los tres modelos en su estado puro (Weber, 2004). Así, el tipo ideal solo funciona como un recurso de método para el análisis de las realidades de la escuela y las prácticas pedagógicas de los docentes en la actualidad. 


\section{Metodología}

De acuerdo con la Secretaría de Educación Municipal (SEM) de Cali, existen alrededor de 1440 establecimientos educativos, 91 de ellos son instituciones educativas oficiales. De las más de 1350 instituciones educativas particulares, alrededor de 214 estaban en 2016 en proceso de cierre temporal o definitivo. El anuario registra que la matrícula oficial "representa más de dos tercios del total de la población (...) con un total de 240.240 estudiantes equivalentes al $63,77 \%$ del total de la matrícula. La matrícula privada con una cantidad de 136.470 estudiantes representa el $36,23 \%$ del total" (SEM, 2016).

Para abordar los propósitos de este estudio se realiza un ejercicio de estadística descriptiva, apoyada en una base de datos del ICFES para más de 500 mil estudiantes que presentaron las Pruebas Saber 11 a nivel nacional en 2016, filtrando información para alrededor de 22700 estudiantes que presentaron las mismas pruebas por una IEC. Se eliminaron aquellos casos que no eran estudiantes de Cali o que hicieron la prueba de forma individual. Esta base de datos tiene información sobre rasgos demográficos, socioeconómicos, el ambiente escolar del hogar y algunas características del plantel educativo.

A través de un muestreo no probabilístico se aplicó una encuesta en 46 IEC a más de 130 docentes sobre sus prácticas pedagógicas y didácticas. Se pudo establecer que en estas IEC encuestadas fueron valorados alrededor de 2968 estudiantes en 2016. De igual forma, se revisó el modelo pedagógico de estas IEC; ello para procurar establecer lineamientos institucionales para las prácticas pedagógicas activas - desde enfoques de la educabilidad- $u$ otras pedagogías donde se expresan estrategias más tradicionales - desde enfoques de la enseñabilidad- 0 que en la práctica combinaban estos enfoques.

De forma específica, las variables exploradas se relacionan con la finalidad que se le asignaba al acto educativo, el lugar de los contenidos, los roles asumidos en el acto educativo por docentes y estudiantes, las estrategias y metodologías de trabajo, las secuencias didácticas y, por último, el lugar y sentido de la evaluación. La forma como cada docente actúa visibilizaba una orientación y una huella pedagógica en su quehacer que se orienta hacia modelos más hetero, auto e interestrucurantes o una combinación de ellos.

\section{Resultados \\ en las Pruebas Saber 11}

Al observar los resultados en las Pruebas Saber 11, se revelan unas desigualdades significativas según el departamento donde se realizó el examen. La prueba fue presentada por 548110 estudiantes a nivel nacional, de los cuales $16,6 \%$ se encuentran en Bogotá, seguidos de Antioquia y Valle del Cauca con $13,3 \%$ y $7,4 \%$, respectivamente, que junto al $6,5 \%$ de Cundinamarca, aglutinan al 43,7 \% del total de estudiantes en 2016.

La media nacional de la prueba gira en torno a los 258,24 puntos, magnitud que solo alrededor de 10 departamentos - de los 32 existentes- alcanzan y superan, siendo Bogotá el espacio con el promedio más alto del país con 277,48 puntos, seguido de Boyacá y Santander con 270,65 y 269,93 , respectivamente. Amazonas, Chocó y Vaupés presentan los resultados más precarios en las pruebas con $228,17,220,11$ y 219,94 puntos, respectivamente. Diferencias que expresan las desigualdades y la calidad educativa que se oferta por regiones. 


\section{Resultados Saber 11 en Cali}

En el puntaje global en Saber 11 en Cali, los estudiantes alcanzan en promedio 267 puntos, alrededor de 9 puntos más que la media nacional. Ahora, estos resultados en términos del género, evidencian que si bien los hombres componen el $43,7 \%$ de los estudiantes valorados, alcanzan un desempeño de 274 puntos en promedio, 11 puntos más que los hombres a nivel nacional; magnitud que para las mujeres denota un menor desempeño, con 262 puntos; aunque estas mejor que las mujeres a nivel nacional (254). Así, se evidencia que los hombres tienden a tener mejores resultados dado que solo el $34,9 \%$ se ubica en el rango más bajo ( 0 - 252 puntos), en el cual se encuentran el $44,1 \%$ de las mujeres. En contraste, los hombres tienen una mejor representación en los resultados que van de 253 a 378 puntos (62\%); en este mismo nivel se encuentran el 53,9\% de las mujeres (Figura 1).

En cuanto a las condiciones socioeconómicas, algunos estudios sugieren que los estudiantes de estratos medio y alto evidenciaran mejores resultados, comparados con los alumnos que pertenecen a estratos inferiores (Moncayo, 2016). Este patrón se sostiene en las pruebas de 2016, al punto que los estudiantes que proceden del estrato 1 alcanzan en promedio 245 puntos, que suben para los estratos 2 y 3 a 258 y 276 , respectivamente; estos tres estratos concentran el $85,6 \%$ de los estudiantes valorados en este periodo. Sin embargo, es en los estratos 4, 5 y 6 donde se pasa la barrera de los 300 puntos, logrando en el estrato 6 alrededor de 345 puntos en promedio, casi 100 puntos más que el estrato 1. De esta forma se revela cómo las condiciones de vida, la disposición de capitales, manifiestan la probabilidad de obtener mejores resultados en las Pruebas Saber. Esto también se relaciona con que, a mayores carencias económicas, "mayor es la probabilidad de que los padres muestren desinterés por las tareas académicas de los hijos y como consecuencia no estimulen, ayuden, ni motiven al alumno" (Moncayo, 2016, p. 9).

Figura 1

Puntaje total en las Pruebas Saber 11, según el género de los estudiantes.

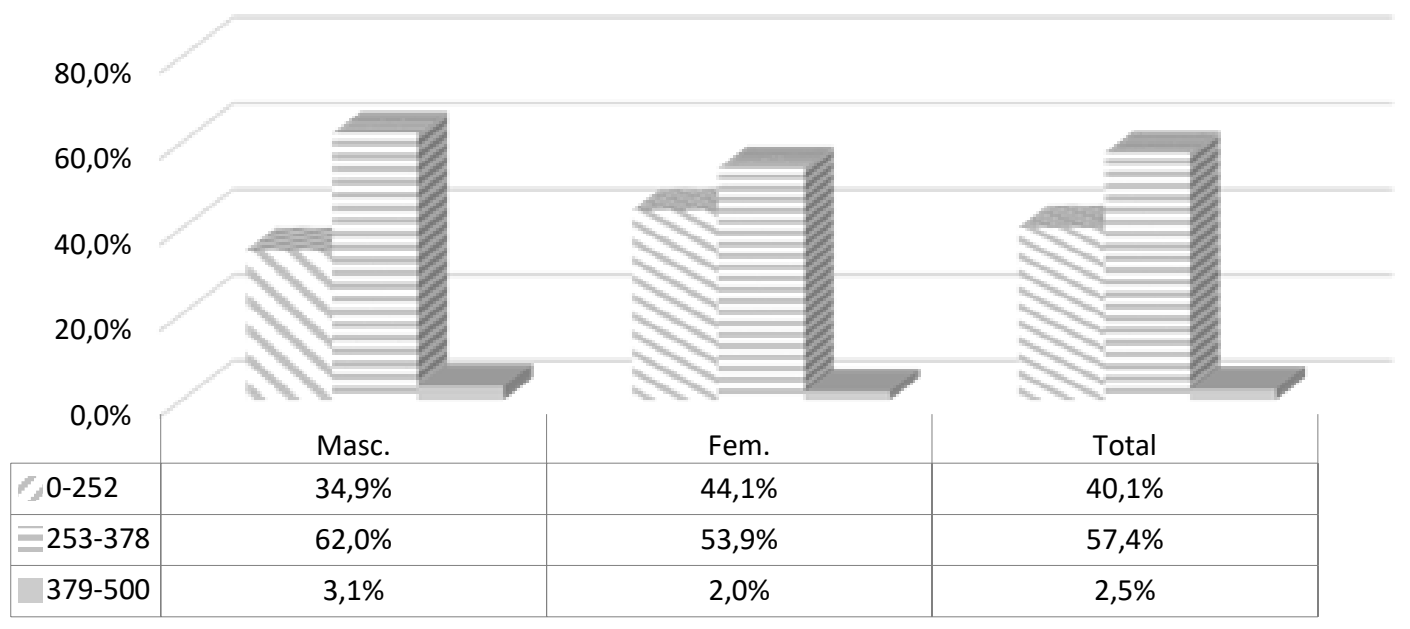

Fuente: Elaboración propia, con base en ICFES (2016). 
El Sisbén es un programa social del gobierno en Colombia, y funciona como una variable proxi para conocer las condiciones de existencia de los estudiantes. En este sentido, un $28,3 \%$ de los evaluados que estaban clasificados en el nivel I alcanzan en promedio 245 puntos, en nivel II estaban el 14,3\% de los estudiantes, logrando 254; y en nivel III un $1,9 \%$ con 264 puntos. Mientras, aquellos estudiantes que no están clasificados en el Sisbén obtienen alrededor de 282 puntos en promedio y representan alrededor del $53 \%$ de la población valorada.

Igualmente, existe un efecto negativo de las horas laboradas por el estudiante, dado que esto afecta las actividades académicas y el rendimiento (Moncayo, 2016). En efecto, un $90,9 \%$ de los estudiantes que afirmaban no trabajar consiguen alrededor de 269 puntos en las Pruebas Saber 11, 28 puntos más que aquellos estudiantes que laboran 20 horas 0 más a la semana, aunque estos representan solo el 2,5\% de la población.
Ahora bien, "es importante destacar que el nivel educativo de los padres, la renta familiar y la presencia de un ambiente estimulante en el hogar suele ir asociado al buen rendimiento académico de los hijos" (Moncayo, 2016, p. 9). Así, el ambiente educativo del hogar es un referente significativo para entender estos resultados Saber 11 . Un $68 \%$ de los estudiantes que proceden de hogares donde ninguno de los dos padres tiene estudios a nivel superior - nivel bajo - alcanzan alrededor de 253 puntos en promedio; mientras, el $17,6 \%$ de los estudiantes proceden de hogares donde ambos padres tienen formación a nivel superior -nivel alto-, y obtienen alrededor de 311 puntos, 57 puntos más en promedio que los primeros (Figura 2).

El puntaje total en las Pruebas Saber 11, según el nivel educativo de los padres, demuestra que un $49,6 \%$ de los estudiantes con padres que no tiene estudios a nivel superior -bajo nivel- logran 252 puntos o menos en las pruebas; a diferencia de los que cuentan con padres profesionales, que registran un

\section{Figura 2}

Puntaje total en las Pruebas Saber 11, según el nivel educativo del hogar de los estudiantes.

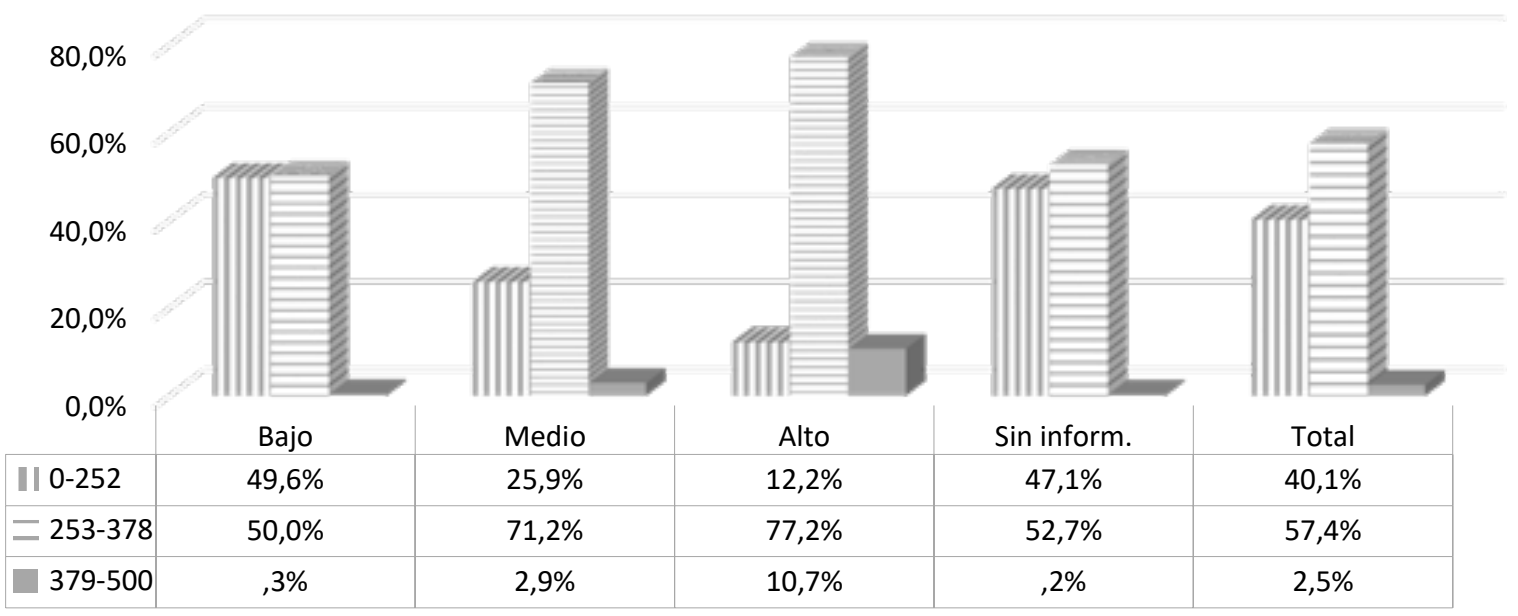

Fuente: Elaboración propia, con base en ICFES (2016). 
$12 \%$ de bajos resultados. Por el contrario, los estudiantes con padres profesionales - nivel alto- tienen una representación mucho más importante en los desempeños más altos, casi un $11 \%$ logran puntajes iguales o superiores a 379 (Figura 2).

En cuanto, al "efecto escuela", las condiciones socioeconómicas en el hogar aumentan la probabilidad de obtener mejores resultados en las Pruebas Saber 11, dado que pueden "comprar" una educación de mejor calidad. Ilustrativo de esta dinámica es el hecho de que un $66 \%$ de los estudiantes que no pagaban pensión alcanzaron 256 puntos en promedio; en contraste, para un 7,6 \% de los estudiantes que pagaban de pensión menos de $\$ 87.000$, característico de la oferta educativa privada de menor calidad, obtienen los puntajes más bajos con alrededor 245 , en promedio. Para el rango de pensión entre 120000 y menos de 150000 (3,9\% de los estudiantes) los resultados aumentan significativamente a 290 puntos, y el $10,4 \%$ de los estudiantes que pagaban una pensión de 250000 o más, obtienen en promedio 327 puntos, más de 70 puntos que aquellos que no pagaban pensión.

Esto hay que entenderlo en el contexto de una oferta educativa segmentada en la ciudad y por tanto de calidad desigual. En consecuencia, se configuran unos clusters de IEC, en donde los resultados más bajos en las pruebas se visibilizan especialmente en el oriente de la ciudad -comunas 13, 14, 15, 16 y $21-$ y al occidente, en la zona de ladera -comunas 1,18 y $20-$, de donde proceden alrededor de un $35 \%$ de los estudiantes, consiguiendo en promedio 246 y 262 puntos, respectivamente, teniendo en cuenta que estos espacios presentan las situaciones de vida más desfavorables y las condiciones educativas más precarias, que implican reconocer un efecto del barrio sobre los resultados Saber
11 (Rozo, 2017). Esto difiere de los desempeños alcanzados por las comunas del sur -17 , 19 y 22 - en donde se logran al rededor de 303 puntos en promedio en las pruebas, con un $15,2 \%$ de los estudiantes valorados en el periodo.

Ahora, como se señaló en líneas precedentes, los estudiantes que asisten a escuelas con única jornada tienen mejores resultados (Castro et al., 2016). En nuestro caso se evidencia que el $13,7 \%$ de los estudiantes que proceden de instituciones jornada completa, logran alrededor de 307 puntos, a diferencia de un $5 \%$ y un $2,5 \%$ de los estudiantes que proceden de colegios nocturnos y sabatinos, en donde en promedio en las pruebas tienen puntajes de 220 y 213 . A su vez, el $62,6 \%$ de los estudiantes que cursan sus estudios en la jornada de la mañana tienen en promedio 268 puntos. Un $3 \%$ de las IEC están registradas como bilingües, y logran alrededor de 330 puntos en promedio, a diferencia de la mayoría, que obtienen 266 puntos en estas pruebas. Por último, al observar la naturaleza del establecimiento encontramos que el $51 \%$ de los estudiantes valorados que proceden de un colegio no oficial obtienen en promedio 277 puntos; mientras, las IEC oficiales consiguen alrededor de 10 puntos menos en promedio en las Pruebas Saber 11.

\section{Resultados de las pruebas en Cali}

En la valoración Saber 11 se evalúan cinco pruebas: Lectura Crítica, Matemáticas, Ciencias Naturales, Ciencias Sociales y Ciudadanas, e Inglés. De forma específica, prestamos especial atención a los desempeños logrados por los estudiantes agrupados por el ICFES en cuatro niveles (insuficiente, mínimo, satisfactorio y avanzado). Para ello, contrastamos los resultados de las pruebas Saber 11 en 2016 y las descripciones cualitativas que aporta el 
ICFES, que corresponden a cada uno de estos niveles de desempeño, para entender las competencias desarrolladas por los estudiantes (ICFES, 2018).

En la Figura 3 se presenta el nivel de desempeño individual alcanzado por los estudiantes en cada una de las cuatro pruebas en 2016. En Lectura Crítica se revela que la tercera parte de los estudiantes alcanzan desempeños insuficientes y mínimos en las pruebas del 2016 (Figura 3); según la guía de orientación ICFES se trata de estudiantes que expresan cualidades, habilidades y conocimientos que les permiten identificar elementos literales en textos continuos y discontinuos sin establecer relaciones de significado o que alcanzan a comprender estos textos de manera literal. En contraste, los resultados en 2016 revelan que el $65 \%$ de los estudiantes alcanzan desempeños satisfactorios y avanzados; es decir, según las cualidades definidas por el ICFES, son estudiantes competentes para comprender e interpretar información de textos, inferir contenidos implícitos, reconocer estructuras, estrategias discursivas y juicios valorativos. De igual manera, el ICFES afirma que estos estudiantes consiguen dar cuenta de elementos paratextuales significativos presentes en el texto, valorando la posición propia.

En Matemáticas, un $8,1 \%$ de los estudiantes logran desempeños insuficientes (Figura 3); según el ICFES, son estudiantes que son competentes para leer información puntual relacionada con situaciones cotidianas y presentada en tablas o gráficas con escala explícita, cuadrícula; pero pueden tener dificultades al comparar distintos conjuntos de datos, involucrar diferentes variables 0 analizar situaciones alejadas de su vida diaria. Si sumamos el $36,8 \%$ de los estudiantes con desempeños mínimos, llegan casi al $45 \%$ con estos desempeños en las Pruebas Saber 11 en 2016. Por el contrario, un grupo importante de los estudiantes se ubican en un desempeño satisfactorio (46,5\%) en 2016 (Figura 3); la guía

\section{Figura 3}

Nivel de desempeño individual en cada una de las pruebas en las pruebas Saber 11 en 2016.

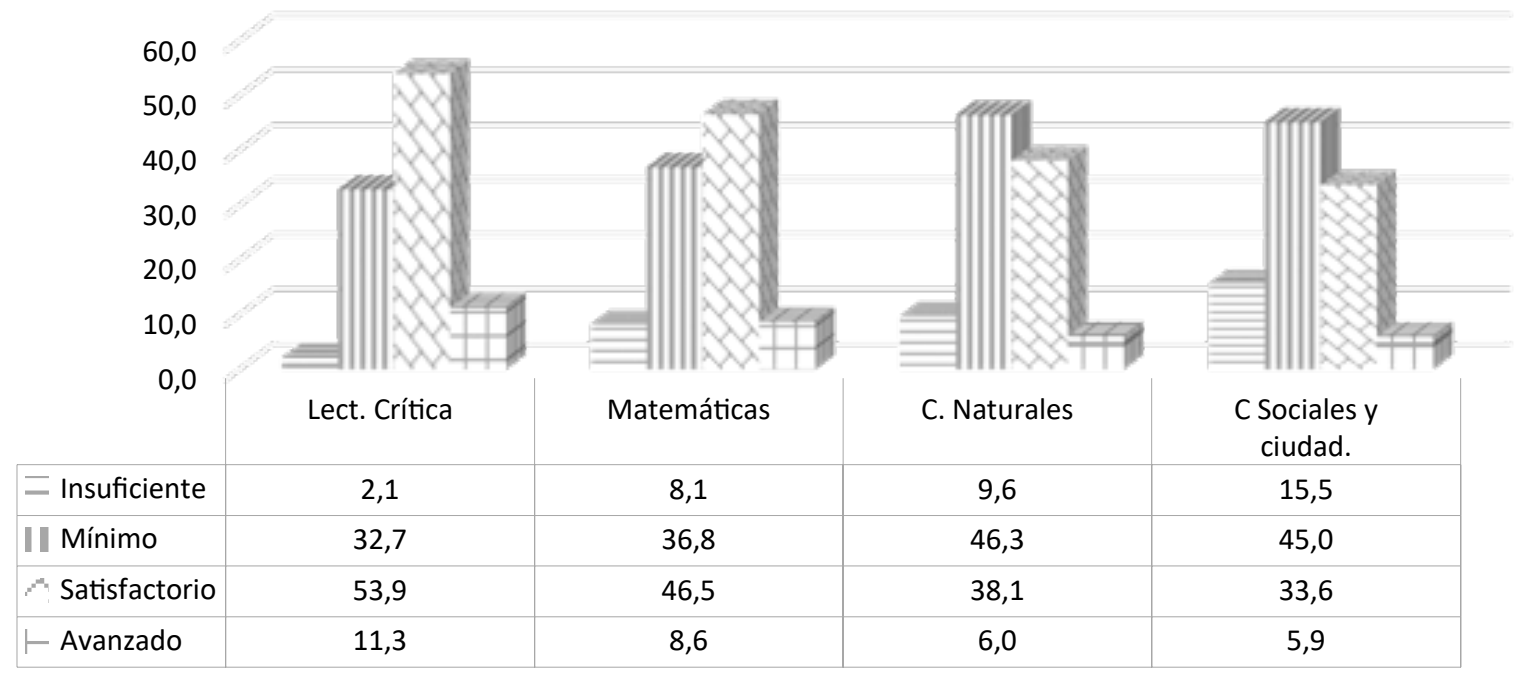

Fuente: Elaboración propia, con base en ICFES (2016). 
ICFES afirma que estos estudiantes describen cualidades, habilidades y disposiciones que les permiten seleccionar información, señala errores y hacer distintos tipos de transformaciones y manipulaciones aritméticas y algebraicas sencillas; esto para enfrentarse a problemas en contextos laborales u ocupacionales, matemáticos o científicos, y comunitarios o sociales. Y, por último, un $8,6 \%$ de los evaluados alcanzan un nivel avanzado en la Prueba Saber 11 de 2016; según el ICFES, son estudiantes que logran resolver problemas que requieren el uso de conceptos de probabilidad, propiedades algebraicas, trigonométricas (ICFES, 2018).

En cuanto a los resultados en Ciencias Naturales, se evidencia una mayor proporción de estudiantes que logran desempeños insuficientes y mínimos en relación con las pruebas de Lectura Crítica y Matemáticas; un $56 \%$ tienen un desempeño mínimo o insuficiente en 2016 (Figura 3). En consecuencia, se relaciona con estudiantes que demuestran un insuficiente o mínimo desarrollo de la competencia indagación; el ICFES les reconoce otras cualidades como identificar información suministrada en tablas, gráficas y esquemas de una sola variable independiente, y la asocia con nociones como tiempo, posición, velocidad, imantación y filtración propios de las ciencias naturales. En contraste, la Figura 3 también revela que solo un $6 \%$ de las personas que respondieron a esta prueba consiguen un nivel avanzado; que según la cualificación establecida por el ICFES son sujetos que usan conceptos, teorías o leyes en la solución de problema que involucran procedimientos, habilidades, conocimientos y un lenguaje propio de las ciencias naturales (ICFES, 2018).

En la prueba de Ciencias Sociales y Ciudadanas, un $60 \%$ de los 22 mil estudiantes valorados en 2016 tienen desempeños insuficientes o mínimos (Figura 3). En términos del ICFES, se adquieren conocimientos y habilidades para reconocer derechos ciudadanos en situaciones sencillas; igualmente, son estudiantes que reconocen deberes del Estado colombiano; identificando relaciones entre las personas y sus cosmovisiones, contextualizan fuentes y procesos sociales, situaciones cercanas a la cotidianidad del estudiante o de conocimiento y amplia discusión pública. En el otro extremo se ubican el $38,1 \%$ de los estudiantes en el nivel satisfactorio. La guía ICFES expresa como las cualidades que representan mejor a estos sujetos como competentes para establecer prejuicios 0 intenciones contenidos en una afirmación y reconocen las dimensiones e intereses involucrados en un problema 0 alternativa de solución. Estas habilidades y conocimientos se valoran tanto en situaciones cotidianas como en contextos más lejanos y complejos. Por otro lado, las Pruebas Saber 11 en 2016 revelan que el nivel avanzado es alcanzado por el 5,6\%. La guía describe que se trata de estudiantes que serían capaces de reconocer algunas disposiciones de la Constitución Política que posibilitan la participación ciudadana y el control a los poderes públicos (ICFES, 2018).

Para la prueba de inglés se evalúan competencias alineadas con el Marco Común Europeo de Referencia (MCER) para las lenguas. Esta prueba clasifica a los evaluados en cinco niveles de desempeño: $A, A 1, A 2, B 1$ y $B+$. Existe población que se encuentra por debajo del primer nivel del MCER, lo cual ha llevado a incluir en la prueba un nivel inferior que corresponde a los mínimos que involucran el manejo de vocabulario y estructuras básicas (A). De igual forma, se incluye un nivel superior al B1 para aquellos estudiantes que superan lo evaluado en este nivel, denominado $B+$. 
En cuanto a los resultados, encontramos que en el nivel A se ubica el desempeño de 1 de cada 3 de estudiantes (33,3\%) en las Pruebas Saber 11 en 2016; siendo estudiantes que no superan las preguntas de menor complejidad de la prueba. En el siguiente desempeño (nivel Al) están el 32,8 \% de los estudiantes valorados en este mismo periodo. Desempeños que el ICFES cualifica como competentes para comprender y utilizar expresiones cotidianas de uso muy frecuente, así como frases sencillas destinadas a satisfacer necesidades inmediatas; y, por último, puede relacionarse de forma elemental con su interlocutor siempre que este hable despacio y con claridad (ICFES, 2018).

En contraste, los datos analizados revelan que un $19,2 \%$ de los estudiantes logran un desempeño A2 en 2016. El ICFES cualifica a estos estudiantes en este nivel como capaces de comprender frases y expresiones de uso frecuente; que describen en términos sencillos aspectos de su pasado y su entorno, así como sus necesidades inmediatas. En relación con el nivel $B 1$, es alcanzado por alrededor del $10,5 \%$ de los estudiantes en 2016; es decir, según el ICFES, comprenden textos, ya sea en situaciones de trabajo, de estudio 0 de ocio. Finalmente, en el desempeño más alto tenemos solamente el $4,5 \%$ de los estudiantes que superan las preguntas de mayor complejidad de la pruebas de inglés en 2016.

\section{Prácticas pedagógicas docentes en Cali y resultados Saber 11}

Un aspecto importante para entender los resultados de las Pruebas Saber 11, se relaciona con el denominado "efecto escuela". Además, de los factores propios de las IEC, emergen otras dinámicas institucionales como las prácticas pedagógicas de los docentes que inciden en el logro académico de los estudiantes:

En promedio, los(as) estudiantes de profesor(as) que dijeron emplear la exposición magistral un $50 \%$ o más del tiempo de clase, tienen 0.41 puntos menos en sus notas finales que los (las) estudiantes de profesores(as) que dijeron emplear otro tipo de técnicas, como exposición magistral con diálogo, discusión grupal, exposiciones de estudiantes, trabajo en equipo, etc., un $50 \%$ o más del tiempo de la clase. (Montero et al., 2007, p. 5)

En la Tabla 1 se observa la distribución de las prácticas pedagógicas, según la zona de la ciudad de las IEC, revelando que las prácticas pedagógicas activas tienen mayor presencia en las comunas del sur (62\%); mientras, en la zona oriente se concentran las IEC con otras prácticas pedagógicas, más próximas a la clase magistral, la memorización y la receptividad $(60 \%)$.

Tabla 1

Zona de la ciudad ${ }^{3}$, según tipo de pedagogías implementadas en la IEC.

\begin{tabular}{lrrr}
\hline Zona & $\begin{array}{c}\text { Pedagogías } \\
\text { activas }\end{array}$ & $\begin{array}{c}\text { Otras } \\
\text { pedagogías }\end{array}$ & \multicolumn{1}{c}{ Total } \\
\hline Oriente & $0,0 \%$ & $59,8 \%$ & $33,6 \%$ \\
\hline Sur & $61,8 \%$ & $7,1 \%$ & $31,0 \%$ \\
\hline Norte & $2,2 \%$ & $0,0 \%$ & $1,0 \%$ \\
\hline Centro & $11,8 \%$ & $0,0 \%$ & $5,2 \%$ \\
\hline Eje norte-sur & $14,5 \%$ & $27,8 \%$ & $22,0 \%$ \\
\hline Zona rural & $9,6 \%$ & $5,3 \%$ & $7,2 \%$ \\
\hline Total & $100,0 \%$ & $100,0 \%$ & $100,0 \%$ \\
\hline
\end{tabular}

Fuente: Elaboración propia, con base en ICFES (2016) y encuesta prácticas docentes en Cali. 
En consecuencia con lo anterior, estas zonas coinciden con las dinámicas de mejor calidad de vida de la ciudad, al punto que en la zona oriente existe una gran concentración de estratos más bajos y aglutina una mayor proporción de población vulnerable (Alonso et al., 2007). Así, al examinar esta cuestión por estratos se observa que el $60 \%$ de las IEC con prácticas pedagógicas activas están en estratos 4, 5 y 6 . Mientras, de las IEC que implementan otras pedagogías, el 93,5\% actúan en estratos 1,2 y 3.

Por último, el nivel educativo alcanzado por los padres incide y posibilita la elección de una IEC que implemente pedagogías más activas e innovadoras. Un 61,7 \% de estudiantes que proceden de hogares donde ambos padres tienen formación profesional asisten a una IEC con modelos pedagógicos activos; mientras, el $78 \%$ de los estudiantes que proceden de hogares donde ninguno de los dos padres tiene estudios a nivel superior, cursaron sus estudios en IEC donde se trabaja con otras pedagogías (más tradicionales, magistrales y que privilegian el rol protagonista del docente).

Este ejercicio implica reconocer que las pedagogías activas tienden a coincidir con las IEC en donde las pensiones tienden a ser la más altas, al punto que alrededor del $70 \%$ de aquellas donde se cancelan pensiones iguales o superiores a $\$ 150.000$ tienen prácticas pedagógicas activas; en contraste, de las IEC donde no se pagaba ningún tipo de pensión, el 78,5 \% acuden a otras prácticas pedagógicas, las cuales normalmente son IEC oficiales, otros por concesión o colegios no oficiales que actúan en sectores populares de la ciudad, especialmente en las zonas oriente y occidente de la ciudad, como veíamos en la Tabla 1.
Resultados en las pruebas Saber 11 y prácticas pedagógicas

En cuanto al puntaje total obtenido en las Pruebas Saber 11, según las prácticas pedagógicas implementadas en las IEC observadas, se destaca que solo el $11 \%$ de los estudiantes que se ubican en los rangos menores de desempeño - 252 puntos o menos- proceden de IEC con prácticas pedagógicas activas; mientras, el $51,2 \%$ de los estudiantes que proceden de IEC con otros tipos de pedagogías tienen estos mismos desempeños (Tabla 2).

Tabla 2

Puntaje total Saber 11, según tipo de pedagogías de la IEC.

\begin{tabular}{lrrr}
\hline $\begin{array}{c}\text { Rango de } \\
\text { puntaje total }\end{array}$ & $\begin{array}{c}\text { Pedagogías } \\
\text { activas }\end{array}$ & $\begin{array}{c}\text { Otras } \\
\text { pedagogías }\end{array}$ & Total \\
\hline $0-252$ & $11,9 \%$ & $51,2 \%$ & $34,0 \%$ \\
\hline $253-378$ & $69,1 \%$ & $48,1 \%$ & $57,3 \%$ \\
\hline $379-500$ & $19,0 \%$ & $0,7 \%$ & $8,7 \%$ \\
\hline Total & $100,0 \%$ & $100,0 \%$ & $100,0 \%$ \\
\hline
\end{tabular}

Fuente: Elaboración propia, con base en ICFES (2016) y encuesta prácticas docentes en Cali.

De forma específica, en la Figura 4 se presenta el nivel de desempeño individual de los estudiantes en 2016 para las pruebas de Lectura Crítica y Matemáticas, según el modelo pedagógico que utilizan las IEC. Se revela cómo el $87,7 \%$ de los estudiantes valorados en Lectura Crítica y el 83,4\% valorados en Matemáticas y que proceden de IEC con pedagogías activas, alcanzan niveles de desempeño entre avanzado y satisfactorio.

Por el contrario, los estudiantes que proceden de IEC con otros modelos pedagógicos, en Lectura Crítica y en Matemáticas solo alcanzan el $56,3 \%$ y el $45,8 \%$ en los niveles de desempeño entre avanzado y satisfactorio. 


\section{Figura 4}

Nivel de desempeño individual en lectura crítica y matemáticas en las pruebas Saber 11 en 2016, según modelo pedagógico.

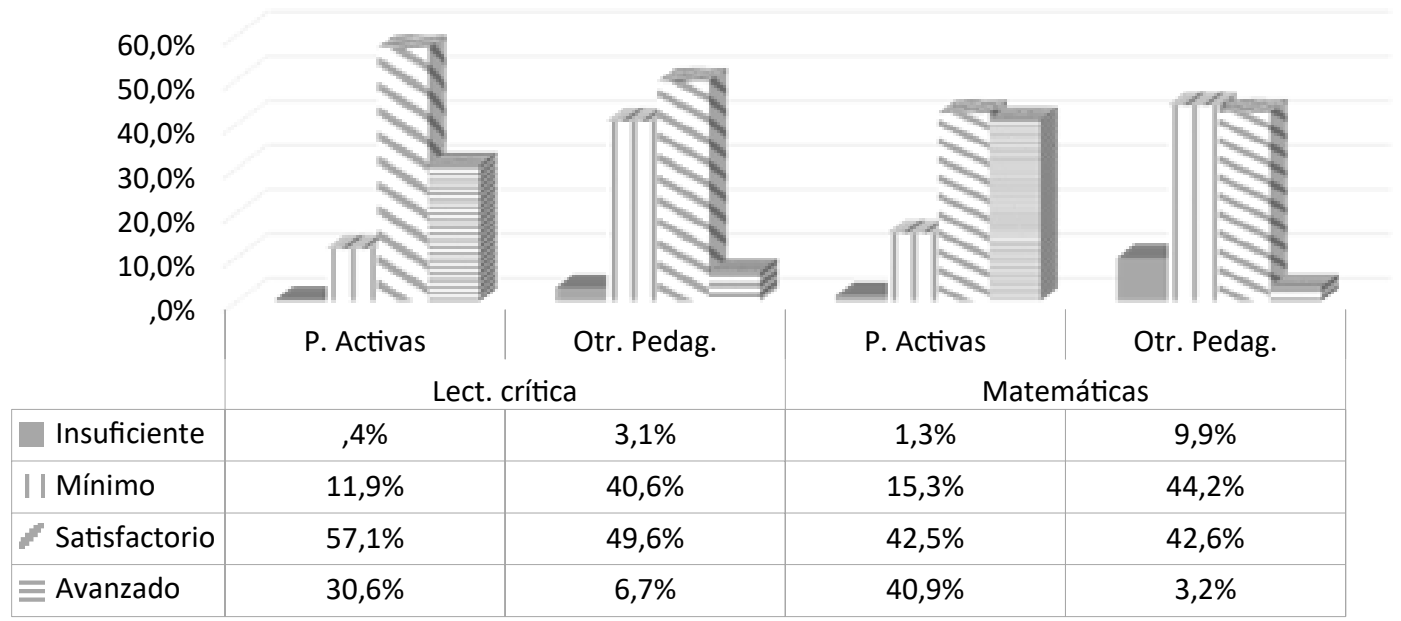

Fuente: Elaboración propia, con base en ICFES (2016) y encuesta prácticas docentes en Cali.

En suma, alrededor de la mitad de los estudiantes que proceden de IEC donde se trabaja desde apuestas pedagógicas no activas o con diferentes combinaciones de las mismas no superan desempeños insuficientes o mínimos.
Para el caso de los resultados en las pruebas de Ciencias Naturales y Ciencias Sociales y Ciudadanas que se recogen en la Figura 5, encontramos que un $78,1 \%$ y un $74,8 \%$ de los estudiantes que provienen de IEC con

\section{Figura 5}

Nivel de desempeño individual en las Pruebas Saber 11 de Ciencias en 2016, según modelo pedagógico.

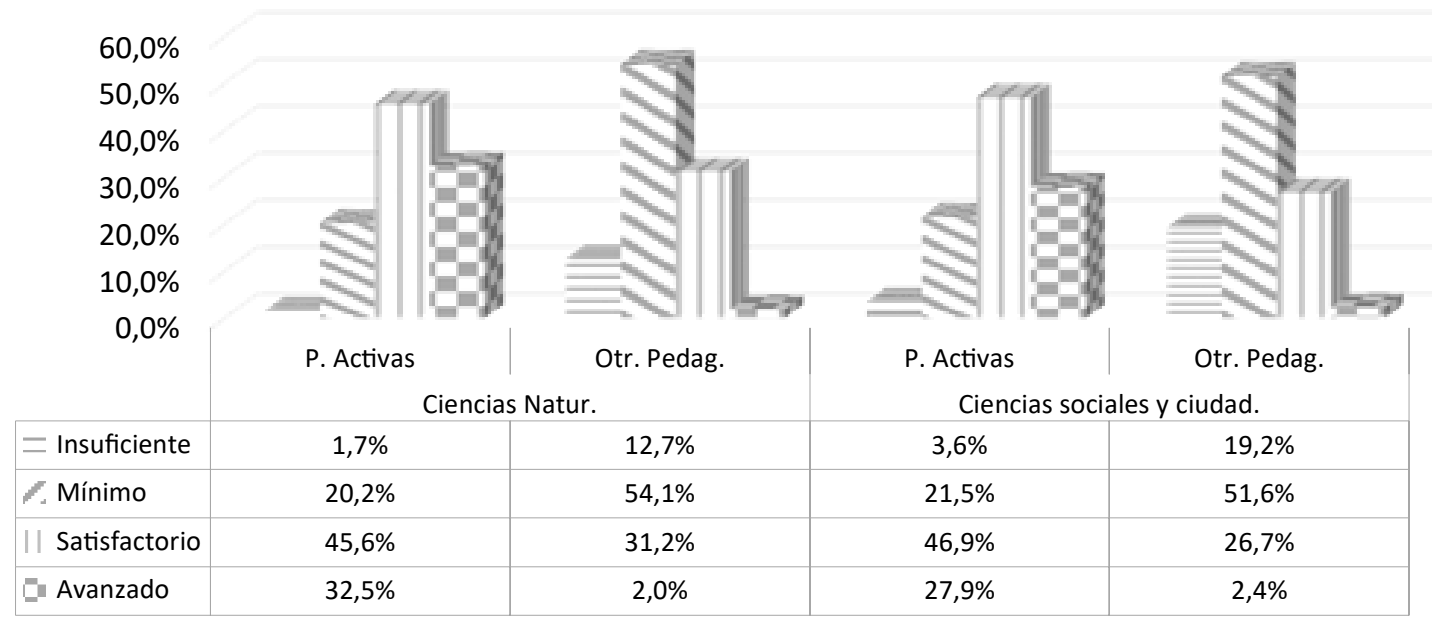

Fuente: Elaboración propia, con base en ICFES (2016) y encuesta prácticas docentes en Cali. 
prácticas pedagógicas activas logran desempeños entre satisfactorio y avanzado; por su lado, la tercera parte de los estudiantes que proceden de colegios con otras prácticas pedagógicas están en estos mismos niveles de desempeño.

Es llamativa la situación que alrededor del $70 \%$ de los estudiantes que proceden de instituciones con otras pedagogías en la pruebas de Ciencias Sociales y Ciudadanas no superan los desempeños insuficiente o mínimo en estas dos pruebas.

\section{Prácticas pedagógicas docentes en Cali}

Como se mencionó en líneas precedentes, se aplicó una encuesta que buscó caracterizar los saberes y prácticas pedagógicas de los docentes de las IEC sobre los sentidos que se le asignan a la educación, el lugar de los contenidos, estrategias y actividades didácticas y, por último, el lugar y los sentidos de la evaluación. Para ello, se les pidió a los docentes que ponderaran la frecuencia con que se efectuaban estas prácticas en una escala, siendo 1 la menor frecuencia y 5 la mayor realización de la misma. A continuación se examinan las medias de estas prácticas.

Al revisar las frecuencias generales con las que los docentes ponen en práctica cada una de estas dinámicas encontramos que en primer lugar aparece la preocupación por la metodología $(3,95)$ luego el sentido o finalidad del acto educativo $(3,93)$, seguido de la inquietud por los contenidos $(3,89)$ y con menos frecuencia se presta atención a la evaluación $(3,73)$.

El sentido o finalidad del acto educativo es un tema central, dado que "tiene que ver con los propósitos, ya que allí se plasman nuestra concepción sobre el individuo y la sociedad, y se delimitan nuestras intenciones educativas" (de Zubiría, 2006, p. 34). En este sentido, la frecuencia con que los docentes valoran las siguientes afirmaciones reflejan el sentido o propósito que le asignan al proceso educativo. De forma general, consideran que el sentido radica en promover el desarrollo integral de los estudiantes $(4,45)$; seguido, dicen que se estructura el conocimiento a partir del diálogo pedagógico entre el docente, el estudiante, los conocimientos y el contexto $(4,31)$; revelando de esta forma prácticas más cercanas a apuestas pedagógicas más activas e interestructurantes. Sin embargo, en las aulas también encontramos docentes que afirman que una finalidad de la educación se relaciona con transmitir el saber cultural acumulado por la humanidad $(4,19)$; otros docentes afirman que el conocimiento se ha generado históricamente por fuera del salón de clase y la tarea del maestro es transmitir ese saber acumulado $(3,40)$; estas últimas prácticas reflejan intereses cercanos a las formas más tradicionales o heteroestructurantes de entender los sentidos de la educación.

En relación con el lugar que tienen los contenidos en el proceso educativo se evidencia que los temas trabajados en clase buscan el desarrollo de formas de aprendizaje significativo, que el estudiante pueda conectar nuevos conocimientos con aquellos que él ya posee $(4,34)$, y que el trabajo realizado en las clases busca que los estudiantes experimenten, construyan y afiancen aprendizajes a través del descubrimiento $(4,27)$; menos frecuente aparecen prácticas que se orienten a la organización de los conocimientos y conceptos en función de la recepción y repetición de un conocimiento ya existente o elaborado $(3,33)$ o que los contenidos procuren fortalecer los aprendizajes de información y la 
memorización de contenidos precisos $(3,04)$. Es llamativo el hecho de que no hubo mayores diferencias entre los profesores que favorecieron la extensión de los temas y aquellos que privilegiaban la profundidad de los temas, con medias en torno a 4.

En cuanto a las estrategias metodológicas y didácticas los docentes evidencian desencuentros en sus apuestas prácticas, si bien algunos afirman que privilegian en sus clases el diálogo de saberes y la acción desarrollada de forma relevante por el estudiante y el docente $(4,42)$, que son prácticas cercanas al modelo interestructurante, también se destacan otras acciones como el desarrollo de estrategias que favorecen la atención del estudiante, la promoción de la disciplina y el seguimiento de las orientaciones docentes como factores claves para el aprendizaje $(4,40)$, que son prácticas más cercanas al modelo heteroestructurante; así mismo, otros favorecen metodologías que permiten vivenciar, operar y experimentar el conocimiento a través del trabajo activo de los estudiantes $(4,40)$, lo cual escenifica prácticas de carácter más constructivista 0 autoestructurante. En relación con las secuencias de los aprendizajes, tal parece que los docentes privilegian en sus metodologías secuencias retrospectivas $u$ otras que se ajusten a las experiencias de aprendizaje $(3,88)$; mientras, otro grupo considera que sus clases también incluyen secuencias cronológicas y acumulativas. Tal vez, en menor medida, existe un grupo de docentes que ponen en práctica didácticas magistrales y expositivas, como apuestas claves para que los estudiantes comprendan los temas propuestos $(3,69)$.

Para terminar, se valora el sentido o lugar que tiene la evaluación. Los docentes le asignan como sentido principal a la evaluación, la promoción de grado o nivel, la capacidad para diagnosticar aprendizajes y contribuir a los procesos formativos $(4,06)$; sin embargo, un grupo de profesores establece como propósito exclusivo la misma promoción del estudiante a otro ciclo o grado de formación -sumativo-, aunque con menor frecuencia $(2,96)$. En relación con la cuestión sobre el quése debe evaluar hay matices y diferencias en las prácticas de los docentes encuestados; si bien es más frecuente combinar estrategias de evaluación que buscan dar cuenta de la formación integral del estudiante en sus distintas dimensiones $(4,33)$, perviven junto a prácticas tradicionales que favorecen la valoración de contenidos precisos y memorizados por el estudiante $(2,36)$. Por último, los docentes consideran importante adelantar una evaluación durante todo el proceso educativo a partir de la experimentación y los sentidos construidos por el estudiante $(4,20)$ y en menor medida se le asigna un lugar a la evaluación de los resultados o producto final del aprendizaje $(3,83)$.

\section{Conclusiones}

A manera de síntesis, se decantan diferencias notables que expresan un crisol de posibilidades entre trayectorias y resultados en las Pruebas Saber 11. En términos geográficos, solo la tercera parte de los departamentos logran tener desempeños superiores a la media nacional, que tienden a agruparse especialmente en los departamentos de mayor concentración urbana y desarrollo económico. Los resultados promedio señalan que los estudiantes de Cali tienen un desempeño superior al comportamiento de los resultados nacionales, lo cual evidencia el efecto que tiene la procedencia geográfica y espacial en los desempeños en las pruebas, dada la disponibilidad de recursos, la oferta cultural y académica de las IEC y de los estudiantes que asisten a estos establecimientos. 
Igualmente, proceder de hogares con padres con mayores niveles educativos incide en los estímulos y la posibilidad de "comprar" o acceder a la diversa oferta de IEC, dado que filtra la población educativa y contribuye a reforzar las desigualdades, según el valor de la pensión, su naturaleza oficial o privada, de baja o alta calidad, infraestructura disponible, personal docente, apuestas pedagógicas, etc.

Estas dinámicas refuerzan la lógica de una oferta desigual y segmentada. Se configuran clusters educativos en algunas zonas de la ciudad, en donde se corresponden las condiciones de vida vulnerables con los puntajes promedio más bajos en las pruebas; y en donde confluyen las IEC privadas de mayor prestigio, bilingües y con pensiones más altas, con pedagogías innovadoras, se revelan mayores posibilidades de mejor desempeño en los estudiantes que pueden "elegir" estas trayectorias o circuitos académicos.

Los resultados para cada una de las pruebas evaluadas señalan importantes diferencias en los desempeños en las pruebas entre sí, denotando mejores logros en la prueba de Lectura Crítica y una situación más preocupante en la prueba de Ciencias Sociales y Ciudadanas. Es llamativo que esta prueba concentre un número significativo de estudiantes con desempeños insuficientes 0 mínimos.

Por último, es complejo establecer modelos pedagógicos puros en la práctica de los docentes, especialmente si trabajan en las instituciones de menor prestigio de la ciudad, evidenciando que estas se configuran como un stock de vivencias y experiencias a los que se acude y se ajusta ante las demandas del entorno de las IEC.
Se evidencia cómo van ganando un lugar significativo las prácticas pedagógicas activas, decantando procesos que procuran innovar y transformar prácticas "tradicionales" centradas en la enseñabilidad, la magistralidad y los contenidos en las IEC; en consecuencia, se revelan apuestas que resignifican los sentidos de la educación más allá de la "reproducción y transmisión" del acervo cultural acumulado por la sociedad. Como vimos, esta dinámica es importante dado que las apuestas pedagógicas de las IEC, en sinergia con otros factores, inciden de forma sustancial en los resultados y desempeños de los estudiantes en las Pruebas Saber 11 del 2016. Situación que tiende a acentuarse, al descentrar los procesos formativos de los contenidos educativos y adelantar apuestas formativas por "Competencias" (ICFES, 2018). Esto abre un campo de reflexión y estudio para examinar con mayor profundidad el alcance de esta relación entre las prácticas pedagógicas y el rendimiento en las Pruebas Saber 11 que permitan reconstruir los ritmos, los sentidos, las prácticas y los logros situados en contexto.

\section{Referencias}

Alonso, J. C., Arcos, M. A., Solano, J. A., Vera, R. y Gallego, A. I. (2007). Mirada descriptiva a las comunas de Cali. Universidad Icesi-Cienfi.

Cárcamo, C. y Mola, J. (2012). Diferencias por sexo en el desempeño académico en Colombia: un análisis regional. Revista Economía \& Región, 6(1), 133-169.

Castro, G., Díaz, M. y Tobar, J. (2016). Causas de las diferencias en desempeño escolar entre los colegios públicos y privados: Colombia en las pruebas Saber 11 2014. Pontificia Universidad Javeriana. 
Ezcurra, A. M. (2011). Igualdad en la educación superior: un desafío mundial. Universidad Nacional General Sarmiento, CONADU.

Flórez, R. (2005). Pedagogía del conocimiento, segunda edición. McGraw-Hill interamericana.

Instituto Colombiano para la Evaluación de la Educación (ICFES) (2016). Resultados agregados de los establecimientos educativos en las pruebas Saber 11, 2016-1. https:// www. icfes.gov.co/ resultados-agregados-2016-1

Instituto Colombiano para la Evaluación de la Educación (ICFES) (2017). Informe Resultados nacionales 2014-2016-2 Saber 11. ICFES.

Instituto Colombiano para la Evaluación de la Educación (ICFES) (2018). Guía de orientación Saber 11 para instituciones educativas. ICFES.

Llanos, C., Fuentes, A., Medina, A. y Prieto, N. (2016). Factores institucionales asociados al logro educativo de las pruebas Saber 11 de Ciencias Naturales en colegios de la Región Caribe. Trabajo de grado de Maestría en Educación, Universidad del Norte.

Martínez, W. y Turriago, A. (2015). Análisis de distribución geográfica y espacial de los resultados de las Pruebas Saber 11, ICFES 2005-2012, Colombia. Cuadernos Latinoamericanos de Administración, XII(21), 39-49.

Meza, D. (2016). Algunos factores que influyen en los resultados de las pruebas estandarizadas y censales. Revista Redipe, 5(3), 136-145. https:// revista.redipe.org/index. $\mathrm{php} / \mathrm{1} /$ article/ view/59/57

Moncayo, M. (2016). Determinantes que influyen en el rendimiento académico: un estudio aplicado para Colombia a partir de las Pruebas Icfes-Saber 11. Trabajo de grado para optar al título de Economista. Universidad de La Salle.
Montero, E., Villalobos, J. y Valverde, A. (2007). Factores institucionales, pedagógicos, psicosociales y sociodemográficos asociados al rendimiento académico en la Universidad de Costa Rica. Relieve, 13(2), 215-234.

Not, L. (1994). Las pedagogías del conocimiento. Fondo de Cultura Económica.

Ríos, C. (2008). Las ciencias de la educación en Colombia, 1926-1954: entre universalismo y particularismo cultural. Grupo Historia de la Práctica Pedagógica en Colombia, 223-267.

Rozo, A. J. (2017). La educación secundaria y sus dos dimensiones. Efectos del barrio y del colegio sobre los resultados Saber 11. Revista de Economía del Rosario, 20(1), 33-69.

Secretaría de Educación Municipal de Cali (SEM) (2016). Anuario Educativo. Observatorio de la Educación de Santiago de Cali.

Tenti, E. (2010). Los que ponen el cuerpo: el profesor de secundaria en la Argentina actual. Educar em Revista, núm. esp. 1, 37-76.

Téllez, M. (2015). Variables asociadas al desempeño escolar. Instituto Latinoamericano de Altos Estudios.

Trujillo, A. J. (2015). Análisis de las prácticas y los estilos pedagógicos de los docentes de la institución educativa Ricabrisa de Tarqui, Huila y su relación con los resultados en las pruebas externas "Saber". [Tesis de Maestría en Educación], Universidad del Tolima.

Vives, M. P. (2016). Modelos pedagógicos y reflexiones para las pedagogías del sur, Boletín Redipe, 5(11), 40-55. https:// bit.ly/31TiVWV

Weber, M. (2004). Economía y sociedad. Esbozo de sociología comprensiva. Fondo de Cultura Económica. https:// bit.ly/ 3GB8S7U

de Zubiría, J. (2006). Modelos pedagógicos: hacia una pedagogía dialogante. Cooperativa Editorial Magisterio. 